\title{
Syphilis in the Pleven region, Bulgaria: a retrospective study
}

\author{
Hristina Victorova Haydudova, ${ }^{1,2}$, Dimitar Konstantinov Gospodinovi,2, Milena Dimitrova Karcheva ${ }^{3 凶}$ \\ ${ }^{1}$ Clinic of Dermatology and Venereology, University Hospital of Pleven, Pleven, Bulgaria. ${ }^{2}$ Department of Dermatology and Venereology, Medical \\ University of Pleven, Pleven, Bulgaria. ${ }^{3}$ Department of Epidemiology, Medical University of Pleven, Pleven, Bulgaria.
}

\begin{abstract}
Introduction: Syphilis is a sexually transmitted infection whose significance to public health has not lost its relevance. Changes in the epidemiology of the disease have been observed in the last decade. This study describes syphilis trends in the Pleven region (Bulgaria).

Methods: Data from 2009 to 2018 were extracted from the National Center for Public Health and Analyses in Sofia and the Regional Health Inspectorate in Pleven and analyzed.

Results: There were 3,602 cases of syphilis during the 10-year period in Bulgaria. The incidence rate (per 100,000) during the period observed varied from 4.2 in 2011 to a maximum rate of 7.5 in 2017. For the same period, 119 cases of syphilis were reported in the Pleven region, with highest incidence rate registered in 2009 (7.2), and the lowest rate registered in 2014 (2.6). The disease was more common in men. All ages are affected, but the largest group is 25 to 29 years old. During the study period, 219 cases of congenital syphilis were registered in the country, three of them in Pleven region.

Conclusions: Retrospective observational data confirmed a rising trend in syphilis cases, especially among younger people. Further study of risk groups and the expansion of promotional activities among adolescents is needed.
\end{abstract}

Keywords: syphilis, epidemiology, Pleven, Bulgaria

Received: 24 February 2020 | Returned for modification: 2 April 2020 | Accepted: 20 April 2020

\section{Introduction}

Syphilis is a very old sexually transmitted infection (STI) whose significance to public health has not lost its relevance today. Although the overall trend in the spread of the disease remains relatively stable, changes in the epidemiology of this disease have been observed in the last decade. There are approximately 6 million cases of syphilis annually worldwide, with the worst-affected population between 15 and 49 years old. The incidence varies depending on the region and the sex of the patient (1). Countries with high-income and stable economies, as well as countries in the European Union, have recently experienced increased rates of syphilis among heterosexuals and men who have sex with men (MSM). Another trend is the increase in cases of congenital syphilis (in the United States and Japan), which is associated with an increased number of infected women (2). These data raise questions such as: Why is the occurrence of syphilis increasing although modern diagnosis and treatment are accessible? What are the medical, social, and economic losses from this trend? What measures are needed to overcome the consequences from the disease? Such consequences are particularly noticeable in low-income countries because they have a high proportion of congenital syphilis and health failures related to it. The past two decades have seen tremendous progress in the prevention of HIV transmission from mother to child. This is not the case with the transmission of syphilis following the same pathway. In 2015, Cuba became the first country to eliminate perinatal HIV and syphilis. This country's experience showed that the goal of double elimination is achievable and feasible with the effective integration of prophylactic programs for both diseases at the same time (3).

Syphilis is a disease that is subject to international surveillance and control. In 2016, the WHO published the strategic document "Global Health Sector Strategy on Sexually Transmitted Infections, 2016-2021" (4). The WHO goal is to achieve a radical decline and end of new STIs, and the morbidity and mortality related to them.

Syphilis is subject to compulsory registration, notification, and reporting in Bulgaria, according to state regulations (5). The cases are classified into the following categories: possible, probable, and confirmed. The cases are reported by centers for dermatological and venereal diseases or by dermatology and venereal departments, by laboratories performing serological diagnostics of syphilis, by centers for transfusion hematology and the National Center for Transfusion Hematology, by every healthcare professional that has discovered and diagnosed syphilis to the corresponding centers for skin-venereal diseases, and by the Regional Health Inspectorates (RHI) and National Center for Public Health and Analyses (NCPHA). Diagnosed cases of syphilis, regardless of their clinical form, are subject to monitoring by an outpatient clinic as required by Bulgarian law (6).

The Pleven District is located in north-central Bulgaria, bordering the Danube River, Romania, and other districts. It is divided into 11 municipalities covering an area of $4,653 \mathrm{~km}^{2}$ with a population of 269,752 (February 2011). The province's capital is the city of Pleven. At the end of 2018 its population was $96,610(7,8)$. Pleven is the location of the Medical University of Pleven and University Hospital of Pleven, with clinics in all major medical fields and research units and with modern diagnostic and therapeutic equipment.

This study analyzes the occurrence rate of syphilis in the Pleven District from 2009 to 2018 and identifies trends in the spread, prevalence, and epidemiological characteristics of the disease. 


\section{Methods}

A retrospective study was conducted to determine the incidence of syphilis in Bulgaria and in the Pleven district. The documentary method was applied. A comparative analysis was carried out using statistical data from NCPHA in Sofia and RHI in Pleven, and annual reports on the work of the Consulting Room on Sexually Transmitted Infections in Pleven. The diagnosis of syphilis was made after performing the following diagnostic tests: screening test (rapid plasma reagin test, RPR) and confirmation with the Treponema pallidum hemagglutination assay (TPHA) and enzyme-linked immunosorbent assay (ELISA) Treponema pallidumspecific IgM/IgG. The study covers the period from 2009 to 2018. Extensive and intensive indicators for data analysis were applied: the number of cases, morbidity (per 100,000), sex, and age distribution of cases reported.

\section{Results}

A total of 3,602 cases of syphilis were registered in Bulgaria from 2009 to 2018 . The morbidity (per 100,000) for this period ranged from 4.2 in 2011 to a maximum level of 7.5 in 2017. The average incidence for the time interval was 5.55 per 100,000. For the same period of time, the highest morbidity in the Pleven District was registered in 2009 (7.2), after which there was a decline to the lowest value of 2.6 in 2014. However, during the last 3 years there was an increase again in occurrence to a level of 6.5 per 100,000, registered in 2018 (Fig. 1).

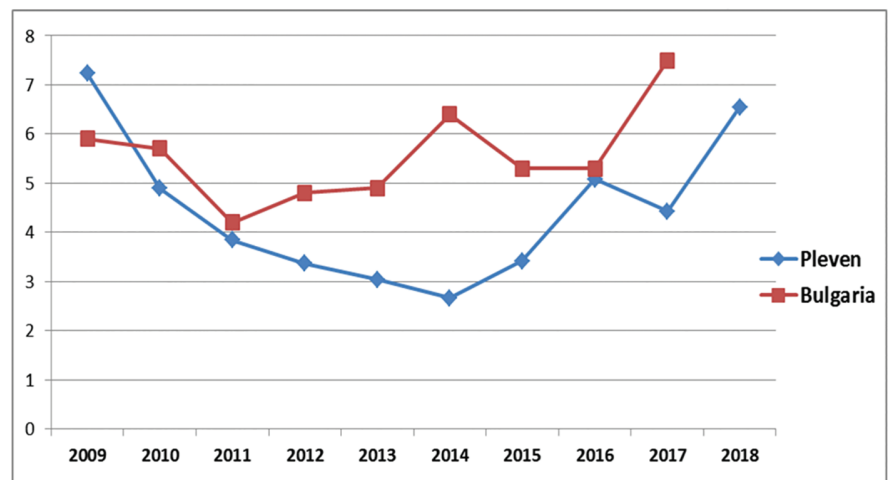

Figure 1 | Incidence rate of syphilis per 100,000 in Bulgaria and the Pleven region, 2009-2018.

There were 119 cases of syphilis registered in the Pleven District from 2009 to 2018, with the sex distribution showing a slight predominance of men: $66(55 \%)$ versus $53(45 \%)$ in women. The distribution of cases by age groups is shown in Figure 2. All ages

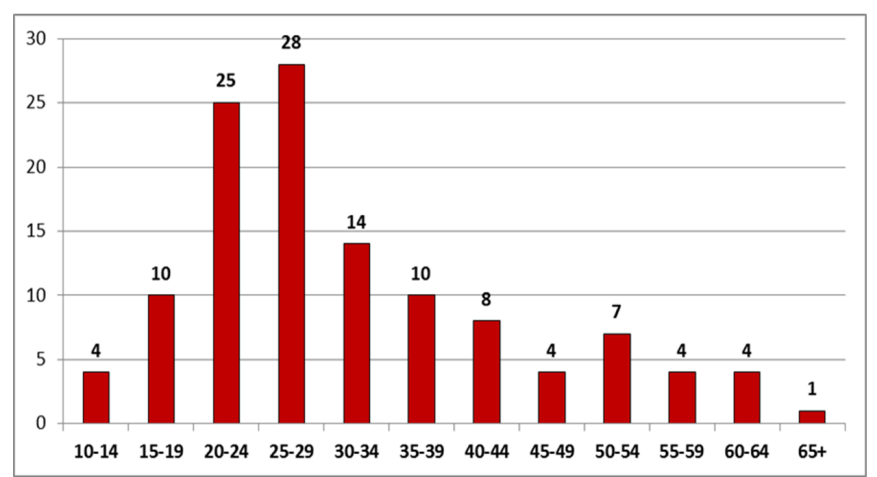

Figure 2 | Reported syphilis cases by age group in the Pleven region, 20092018. are affected, but the largest group is 25 to 29 years old (28 cases), followed by the 20 to 24 age group ( 25 cases).

From 2009 to 2018, a total of 16,807 pregnant women were tested for syphilis in Pleven. Of these women, 293 (1.74\%) were seropositive for syphilis. Three cases of congenital syphilis were registered in the Pleven District from 2009 to 2017, one each in 2012, 2014, and 2015. For the same 9 years, congenital syphilis cases were reported annually in the country, reaching a total of 219 (Fig. 3).

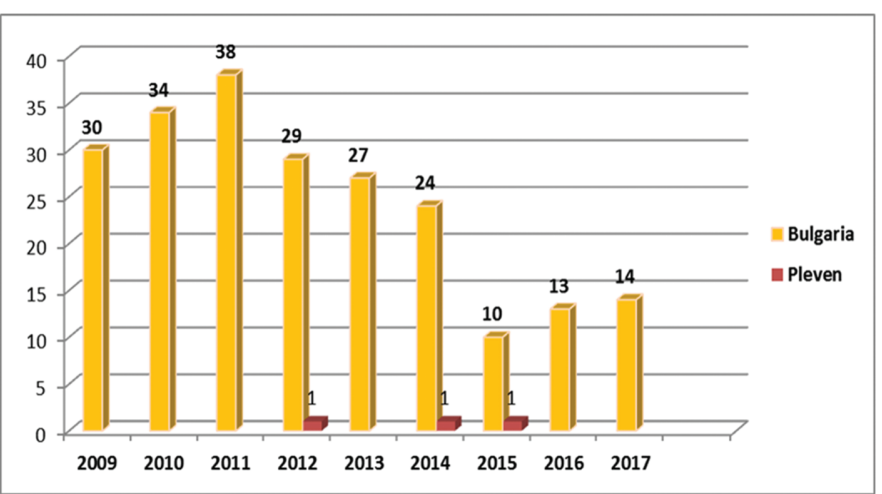

Figure 3 | Reported congenital syphilis cases in Bulgaria and the Pleven region, 2009-2017.

\section{Discussion}

Analysis of the incidence of syphilis in the Pleven District over a 10-year period showed that the highest incidence was at the beginning of the study period ( 7.5 in 2009), followed by a decrease to the lowest, 2.6, in 2014. From 2015 to the end of the period examined, there was a trend of increasing incidence, reaching 6.55 in 2018, a lower value than in the initial year of the study period. This fact shows a general tendency of decreasing syphilis cases in the Pleven District. Comparing the local syphilis rate with the overall incidence in Bulgaria throughout the same period, some differences are found over the years. The country's incidence was 5.9 in 2009, falling to 5.3 in 2010, and even less in 2011, to 4.2, which is the lowest recorded value. Later on, a tendency of increasing morbidity was observed, reaching an occurrence rate of 6.4 in 2014, and 7.5 in 2017, which is the highest value during this study interval. The general tendency in Bulgaria is thus an increasing incidence of syphilis during the study period.

Contrary to the general trend of a decreasing number of syphilis cases in Europe, there is an increase in the incidence rate in certain population groups. According to a report from the European Center for Disease Control (ECDC), the following population groups are identified as being at higher risk: homosexual and bisexual men, prisoners, and drug users. Heterosexuals, HIV-positive persons, migrants, and pregnant women are also described as risky. The syphilis incidence in Bulgaria maintained a stable frequency rate during the time interval studied, with a tendency to increase in recent years (9). A real increase in the incidence rate in Bulgaria was observed after 1990, when major political, social, and economic changes took place.

The syphilis occurrence trend in other European countries is also remaining stable. The lowest morbidity rates were recorded in Croatia (0.7), Portugal (o.8), Slovenia (2.3), Sweden (3.8), Italy (2.7), Estonia (2.6), and Finland (3.2). The highest incidence was reported in Iceland (15.4), Malta (13.5), Spain (10.3), and Germany (9.1), all per 100,000 (2).

The cases we registered showed that both sexes are affected. There was a slight predominance of men throughout the entire pe- 
riod studied. However, slightly more cases of women were registered from 2011 to 2014. The distribution of syphilis cases by sex in Bulgaria shows a slight predominance of men, with the trend from 2017 to an increase in cases among women $(9,10)$. Gender dynamics are also observed in other European countries. The highest occurrence rate in men was reported in 2017 in Iceland (25.0), the United Kingdom (22.0), Malta (22.0), and Germany (17.0). In the same year, the highest incidence among women was observed in Iceland (6.0), Bulgaria (5.4), Latvia (4.8) and Lithuania (4.8) (2).

The analysis of the age distribution of persons registered with syphilis in the Pleven region from 2009 to 2018 shows that all ages are affected. The highest number of cases within age groups is as follows: 28 cases for ages 25 to 29,25 cases for ages 20 to 24 , and 14 cases for ages 30 to 34. A similar trend is observed in other European countries, where the proportion of people affected by age 25 is steadily increasing. These data shows that young people are most vulnerable (11). Studies of reported outbreaks in recent years explain the high incidence in young people with their lifestyles and harmful practices that predispose to the spread of the infection (unprotected sex, drug and alcohol use, HIV-positive status, etc.) $(10,12,13)$. Increasing cases of women of reproductive age also increase the risk of congenital syphilis.

The data from our study show that there are only a few cases of congenital syphilis in Pleven and the surrounding region. However, the total number in Bulgaria is significant and places the country in the leading position among others in Europe. From 2009 to 2017, the incidence of congenital syphilis in Bulgaria varied between 15.2 (2015) and 53.6 (2011) per 100,00o. Cases of congenital syphilis have been registered in 25 European countries in the last 10 years. After Bulgaria, the highest numbers are registered in Portugal, Romania, and Poland. The trend in all reporting countries is a decreasing or unchanged number of cases. Norway, Slovenia, Malta, and Cyprus have no registered cases of congenital syphilis (2). The factors associated with congenital syphilis are risk factors inherent to mothers: high-risk sexual behavior and/or drug use, women deprived of liberty, low income, younger age, factors related to the capacity of the health system to identify and cure syphilis infection during pregnancy, lack of syphilis tests during prenatal visits, inadequate treatment for positive women during pregnancy, and syphilis infection acquired after the first negative screening test $(14,15)$.

The required actions in Bulgaria's Maternal Health Care program include a single test for syphilis for pregnant women during their first visit to a general practitioner or gynecologist. The high number of cases of congenital syphilis in Bulgaria shows that there is insufficient development of counseling and promotion activities for young women and pregnant women. Counseling on all issues of youth and couples before and during pregnancy is necessary to overcome a negative tendency. In a study on syphilis seroprevalence among pregnant women in Bulgaria's Varna District, Tsankova et al. (16) found $0.56 \%(15 / 2,702)$ of the women tested to be seropositive. The only factor that influenced the seropositivity of syphilis was the woman's place of residence: rural women had a significantly higher risk of syphilis infection than those in cities (16).

Identification of risk factors and target groups of the population would improve the timely diagnosis and treatment of women infected with syphilis and would accordingly reduce the incidence of congenital syphilis (17). It is important to note that treatment and monitoring after diagnosed syphilis is carried out by specialists with the necessary qualifications.

\section{Conclusions}

The reported incidence of syphilis in Bulgaria (the average incidence for the time interval considered is 5.55 per 100,000) probably does not correspond to the real one. At the end of the study period there was an increase of the incidence in the Pleven District to 6.5 per 100,000 . The observed trend in the spread of the disease can be overcome by improving the effectiveness of disease control. This can be accomplished by actively searching for patients, adequate treatment, monitoring at outpatient clinics, an individual approach to risk groups in the population, and preventive activities.

\section{References}

1. Peterman TA, Heffelfinger JD, Swint EB, Groseclose, SL. The changing epidemiology of syphilis. Sex Transm Dis. 2005;32:4-10.

2. European Centre for Disease Prevention and Control. Syphilis and congenital syphilis in Europe-a review of epidemiological trends (2007-2018) and options for response [Internet]. Stockholm. [cited 2019 Jul 12]. Available from: https:// www.ecdc.europa.eu/en/publications-data/syphilis-and-congenital-syphiliseurope-review-epidemiological-trends-2007-2018.

3. WHO. WHO validates elimination of mother-to-child transmission of HIV and syphilis in Cuba [Internet]. [cited 2015 Jun 30]. Available from: https://www. who.int/mediacentre/news/releases/2015/mtct-hiv-cuba/en/.

4. WHO. Global health sector strategy on sexually transmitted infections, 20162021 [Internet]. [cited 2016 Jul]. Available from: https://www.who.int/reproductivehealth/publications/rtis/ghss-stis/en/.

5. Ordinance no. 21/18 July 2005 on registration, notification and reporting of communicable diseases, Ministry of Health, Bulgaria [Internet]. [cited 2005 Jul 29]. Available from: https://www.mh.government.bg/bg/search/?q.

6. Ordinance no. 8/3 November 2016 on prophylactic examinations and dispensary, Ministry of Health, Bulgaria [Internet]. [cited 2016 Nov]. Available from: https://www.mh.government.bg/media/filer_public/2016/11/29/naredba8profpregled-dispanserizaciq.pdf

7. Guide-Bulgaria.com. Pleven District [Internet]. Available from: http://bg.guidebulgaria.com/nw/pleven.

8. Republic of Bulgaria, National statistical institute. Pleven District [Internet]. Available from: https://www.nsi.bg/en/content/1146o/district-pleven.

9. Dencheva R, Spirov G, Gilina K, Niagolova D, Pehlivanov G, Tsankov N, et al. Epidemiology of syphilis in Bulgaria, 1990-1998. Int J STD AIDS. 2000;11:819-22.

10. Kabakchieva E, Vassileva S, Kelly JA, Amirkhanian YA, DiFranceisco WJ, McAuliffe TL, et al. HIV risk behavior patterns, predictors, and sexually transmitted disease prevalence in the social networks of young Roma (Gypsy) men in Sofia, Bulgaria. Sex Transm Dis. 2006;33:485-90.

11. Kojima N, Klausner JD. An update on the global epidemiology of syphilis. Curr Epidemiol Rep. 2018;5:24-38.

12. Hart-Malloy R, Rosenthal M, Patterson W, Currenti S, O'Donnell T, Gunn JK. Syphilis among adult males with a history of male-to-male sexual contact living with diagnosed HIV in New York State (excluding New York City): the challenge of intersecting epidemics. PLoS One. 2019;14:e0226614.

13. Dong W, Zhou C, Rou KM, Wu ZY, Chen J, Scott SR, et al. A community-based comprehensive intervention to reduce syphilis infection among low-fee female sex workers in China: a matched-pair, community-based randomized study. Infect Dis Poverty. 2019;8:97.

14. John-Stewart G, Peeling RW, Levin C, Garcia PJ, Mabey D, Kinuthia J. Major infec tious diseases. 3rd ed. Washington, DC: The International Bank for Reconstruction and Development / The World Bank; 2017. Chapter 6, Prevention of motherto-child transmission of HIV and syphilis; p. 113-36.

15. Kirkcaldy RD, Su JR, Taylor MM, Koumans E, Mickey TB, Winscott M, et al. Epidemiology of syphilis among Hispanic women and associations with congenital syphilis, Maricopa County, Arizona. Sex Transm Dis. 2011;38:598-602. 
16. Tsankova G, Todorova T, Kostadinova T, Ivanova L, Ermenlieva N. Seroprevalence of syphilis among pregnant women in the Varna region (Bulgaria). Acta Dermatovenerol Croat. 2016;24:288-90.
17. Korenromp EL, Mahiané SG, Nagelkerke N, Taylor MM, Williams R, Chico MR, et al. Syphilis prevalence trends in adult women in 132 countries-estimations using the Spectrum Sexually Transmitted Infections model. Sci Rep. 2018;8:11503. 